\title{
A sample of new planetary nebulae in the IPHAS and DSH catalogue
}

\author{
Chih-Hao Hsia and Sun Kwok \\ Department of Physics, The University of Hong Kong, Pokfulam Road, Hong Kong, China \\ email: xiazh@hku.hk; sunkwok@hku.hk
}

\begin{abstract}
We report the optical and infrared studies of a sample of five new planetary nebulae (PNe) in the IPHAS and DSH catalogues. The PN status of these objects was confirmed by optical narrow-band imaging and low resolution spectroscopy. One object (IPHASX $\mathrm{J} 195248.8+255359$ ) is found to be a bipolar PN with faint $\mathrm{H} \alpha$ lobes and a bright core in [O III].
\end{abstract}

Keywords. Surveys, planetary nebulae: general, stars: AGB and post-AGB

\section{Introduction}

Although more than $2700 \mathrm{PNs}$ have been detected in our galaxy, the number of Galactic PNs is likely to be be much larger. As the result of dust extinction, many faint Galactic PNs can easily be hidden in the Galactic plane or the Galactic center. Results from the recent $\mathrm{H} \alpha$ survey IPHAS (Drew et al. 2005) offer the opportunity to search for new PNs with low surface brightness. Our work is based on the catalogue of 781 new PN candidates selected from IPHAS (Viironen et al. 2009) and the list of 36 uncatalogued Galactic nebulae observed from DSS and 2MASS surveys (Kronberger et al. 2006).

\section{Observations}

Deep narrow-band images and low-resolution spectra of five PN candidates ( IPHASX J190954.7+120455, DSH J1941.3+2430, IPHASX J194226.1+214522, IPHASX J195248.8 +255359 , and IPHASX J232713.1+650923) were observed at the $2.16 \mathrm{~m}$ telescope of the National Astronomy Observatories of China Xinglong station. The observations were made on the nights of 2010 August $14-16$ with the BFORC $1242 \times 1152$ CCD. The 5 objects were imaged with two narrow-band filters: [O III] $\left(\lambda_{p}=5009 \AA, \Delta \lambda=120\right.$ $\AA)$ and $\mathrm{H} \alpha+\left[\mathrm{N}_{\mathrm{II}}\right]\left(\lambda_{p}=6562 \AA, \triangle \lambda=140 \AA\right)$. For spectroscopic observations, the spectral dispersions of measurements were $4.5 \AA$ pixel ${ }^{-1}$. A slit width of $3.6^{\prime \prime}$ was set to allow greater throughput for weak emissions of faint objects observed in this run.

\section{Results}

The narrow-band images of one (IPHASX J195248.8+255359; PN 4) of the sample objects are shown in Fig. 1. The object shows extended bipolar lobes in the $\mathrm{H} \alpha+[\mathrm{N} \mathrm{II}]$ image. A prominent central dust torus can also seen in the infrared image (Fig. 2). From low resolution optical spectra, our sample nebulae show low-excitation emission lines, suggesting that they are low-excitation PNs. 

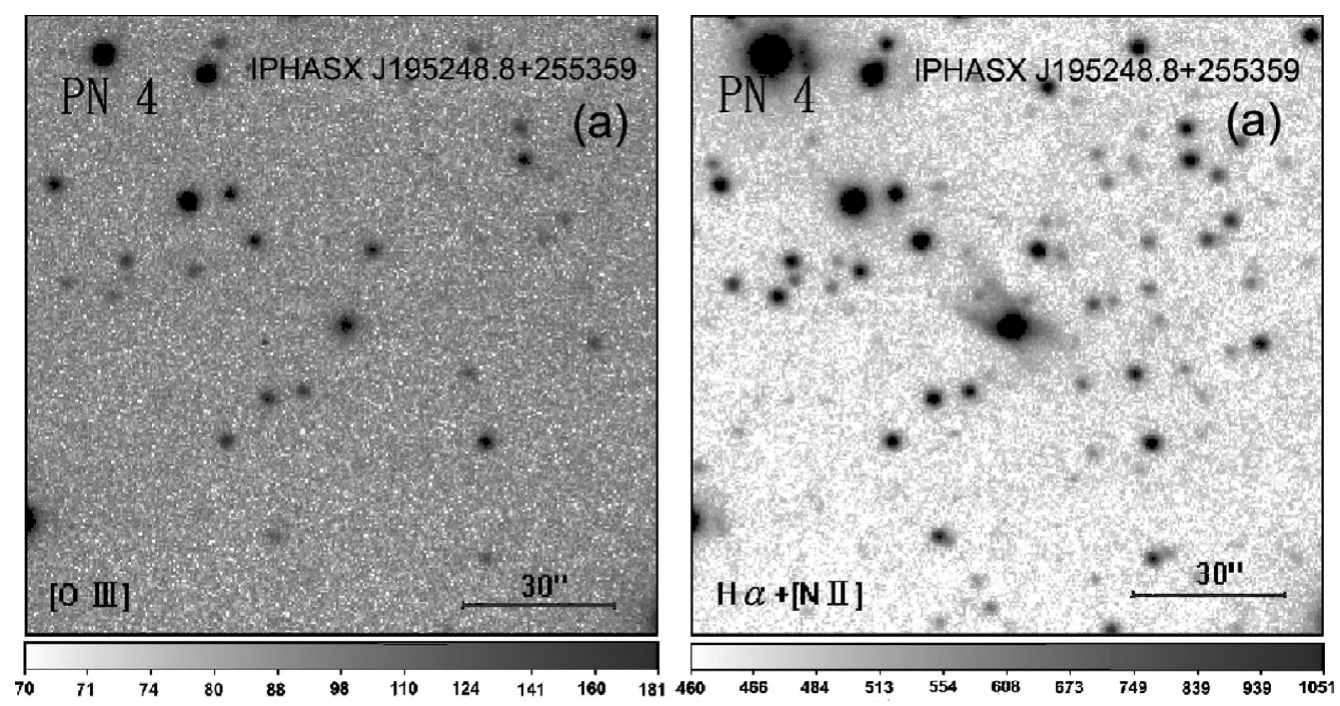

Figure 1. [O III] (left) and H $\alpha+[\mathrm{NII}]$ (right) images of IPHASX J195248.8+255359 (PN 4).

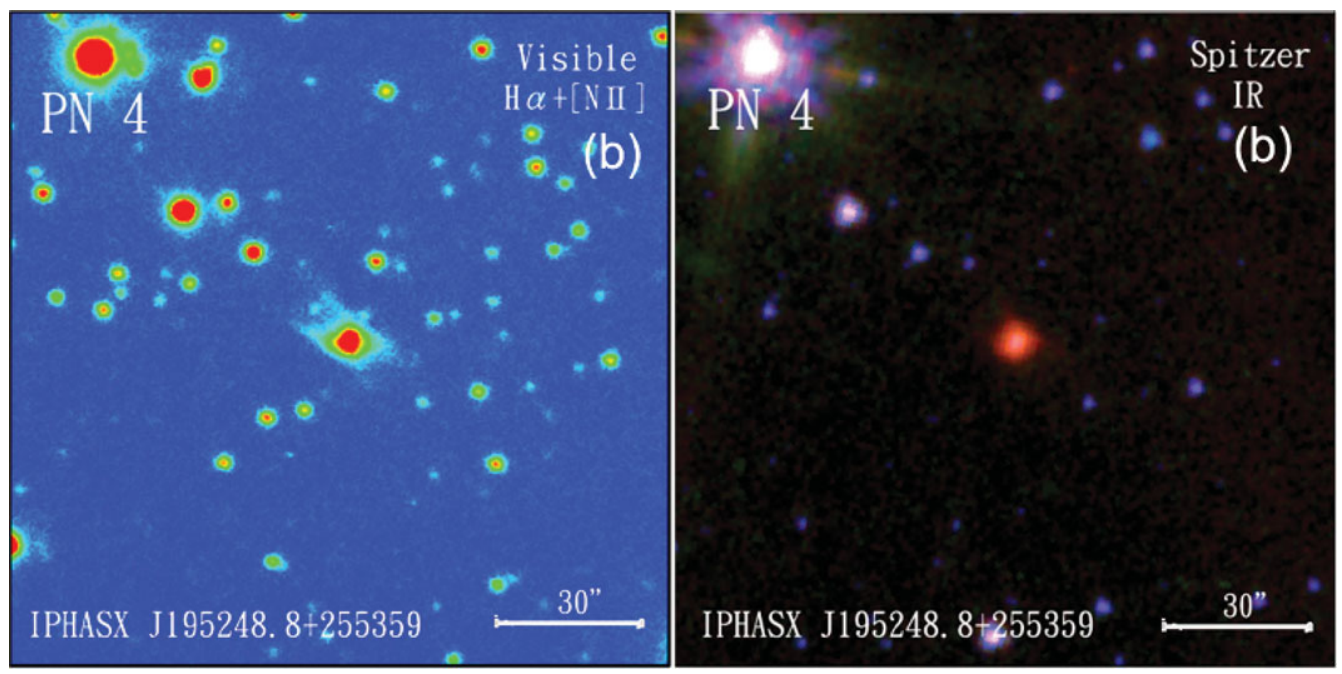

Figure 2. Comparison between the false-color $\mathrm{H} \alpha+[\mathrm{N}$ II] image (left) and color composite of the Spitzer IRAC image (right, blue: $3.6 \mu \mathrm{m}$, green: $4.5 \mu \mathrm{m}$, red: $8.0 \mu \mathrm{m}$ ) of IPHASX J195248.8+255359 .

\section{Acknowledgements}

This work was supported by a grant from the Research Grants Council of the Hong Kong Special Administrative Region, China (Project No. HKU 7031/10P).

\section{References}

Drew, J. E., Greimel, R., Irwin, M. J. et al. 2005, MNRAS, 362, 753

Frew, D. J. \& Parker, Q. A. 2006, IAUS, 234, 49

Kronberger, M., Teutsch, P., Alessi, B. et al. 2006, A\& $\mathcal{G}, 447,921$

Viironen, K., Mampaso, A., Corradi, R. L. M. et al. 2009, A\& A, 502, 113 